\title{
GMZRP: Geography-aided Multicast Zone Routing Protocol in Mobile Ad Hoc Networks
}

\author{
Hui Cheng \\ Internet and Mobile Computing Lab \\ Department of Computing \\ Hong Kong \\ cshcheng@comp.polyu.edu.hk
}

\author{
Jiannong Cao \\ Internet and Mobile Computing Lab \\ Department of Computing \\ Hong Kong \\ csjcao@comp.polyu.edu.hk
}

Xiaopeng Fan

Internet and Mobile Computing Lab

Department of Computing

He Hong Kong Polytechnic University The Hong Kong Polytechnic University
Hong Kong

csxpfan@comp.polyu.edu.hk

\begin{abstract}
This paper presents the design and evaluation of a highly efficient on-demand multicast routing protocol for mobile ad hoc networks (MANETs). The protocol, called Geography-aided Multicast Zone Routing Protocol (GMZRP), eliminates as much as possible duplicate route queries by using a simple yet effective strategy for propagating the multicast route request (MRREQ) packets. GMZRP is the first hybrid multicast protocol taking the advantages of both topological routing and geographical routing. It partitions the network coverage area into small zones and guarantees that each geographic zone is queried only once. GMZRP maintains a multicast forwarding tree at two levels of granularities, the zone granularity and the node granularity. By doing this, it can easily handle route breakage since the zone level information can help recover the link failure at the node level. The results of the performance evaluation of GMZRP using simulation show that, comparing with the well-known multicast protocol ODMRP (On-Demand Multicast Routing Protocol), GMZRP has much lower protocol overhead in terms of query packets and, meanwhile, achieves competing packet delivery ratio.
\end{abstract}

\section{Categories and Subject Descriptors}

D.3.3 [Computer Systems Organization]: ComputerCommunication Networks - network protocols.

\section{General Terms}

Algorithms, Performance, Theory.

\section{Keywords}

Mobile Ad Hoc Networks, Hybrid Routing, Multicast.

\section{INTRODUCTION}

There are mainly two types of routing protocols in MANETs:

QShine 2008, July 28-31, 2008, Hong Kong, Hong Kong. Copyright 2008 ICST ISBN 978-963-9799-26-4 DOI 10.4108/ICST.QSHINE2008.3862 topological routing and geographic routing [1]. In topological routing, mobile nodes utilize topological information to construct routing tables or search routes on-demand. In geographic routing, each node knows its own position and makes routing decisions based on the positions of the destination and its local neighbors.

Group communications in MANETs are important for mobile nodes to work in a cooperative way [2]. Group communications need the support of multicast protocols. A number of ad hoc network multicast routing protocols, using a variety of basic routing algorithms and techniques, have been proposed over the past few years [3, 4]. Similar to unicast routing, multicast routing protocols can also be classified as either topological routing or geographic routing.

Intuitively, by exploiting the advantages of both topological routing and geographic routing simultaneously, more efficient hybrid routing can be developed. Here, hybrid routing refers to the hybrid approach for routing combining both topology driven route optimization and geography driven route optimization. Recently, researchers have proposed a few such hybrid routing protocols [5, 6]. However, they are only for unicast routing. In this paper, we propose GMZRP, which is the first hybrid multicast protocol taking the advantages of both topological routing and geographical routing.

GMZRP operates in an on-demand fashion and utilizes geographic partition to reduce route discovery overhead. The main idea of GMZRP is inspired by the well-known routing protocol ZRP (Zone Routing Protocol) [7], in which a route query is originated at the source and spread throughout the network. ZRP is a unicast routing protocol. In ZRP, a node will receive a lot of duplicate queries due to zone overlapping even under the case that the nodes are uniformly distributed in the network. GMZRP is a multicast protocol and is designed to eliminate duplicate queries.

By partitioning the network coverage area into small zones and guiding the route request packets outward using geographic information, GMZRP can guarantee that each zone is queried only once given an even distribution of the network nodes. In addition, GMZRP extends the unicast route request procedure in ZRP to a multicast tree discovery procedure. To our best knowledge, GMZRP is the first protocol that exploits the symmetrical geographic zone partition in the guidance of the multicast route query. In doing so it discovers the multicast forwarding tree along the shortest paths and with the lowest overhead. 
Another important feature of GMZRP is that it maintains a multicast forwarding tree at two levels of granularities: the zone granularity (the sequential geographic zones that the tree spans in a source routing manner) and the node granularity (the sequential nodes that the tree spans in a hop-by-hop way). At the zone granularity, for each receiver, the source keeps a zone ID chain connecting the source zone to the corresponding receiver zone. An intermediate forwarding node also keeps a zone ID chain connecting its own zone to each downstream receiver zone, which is part of the zone ID chain kept by the source to the same receiver zone. Therefore, at the zone level GMZRP looks like source routing. On the other hand, at the node granularity, the source or an intermediate forwarding node only keeps the information of its child nodes. The multicast packet is forwarded along the multicast tree hop by hop.

The remainder of this paper is organized as follows. Section 2 describes the related work. Section 3 describes the preliminary work including the proposed geographic partition method and the data structures. Section 4 presents the procedure of establishing the multicast tree which is the key component in GMZRP. Section 5 describes the performance evaluation of GMZRP based on extensive simulations, discussing the results in comparison with the well known ODMRP protocol [8]. Finally, Section 6 summarizes the paper and presents the conclusions.

\section{RELATED WORK}

In the following, we introduce ZRP and its two multicast extensions, i.e., MZR [9] and MZRP [10]. However, all these work belongs to topological routing, while the GMZRP protocol proposed in this paper is a hybrid protocol which can solve the duplicate route queries problem by utilizing geographic information.

To our knowledge, ZRP is the first protocol proposing the concept of routing zone. A node's routing zone is defined as a collection of nodes whose minimum hop distance from the node in question is no greater than a parameter referred to as the zone radius. Each node maintains its own routing zone and proactively maintains routes to destinations within its routing zone. An important consequence is that the routing zones of neighboring nodes overlap [7].

In ZRP, when a node bordercasts a route query, the node's entire routing zone is effectively covered by the query. However, since neighboring routing zones heavily overlap, excess route query traffic will be generated as a result of query messages returning to covered zones. Query control mechanisms are proposed to reduce route query traffic by directing query messages outward from the query source and away from covered routing zones, as shown in Figure 1. In [7], ZRP has been enhanced with a collection of query control mechanisms to generate less control traffic than purely proactive route information exchange or purely reactive route discovery do. The query control mechanism includes Query Detection (QD1/QD2), Early Termination (ET), and Random Query Processing Delay (RQPD). However, since all these mechanisms are based on topological information, they cannot solve the overlapping problem completely.

ZRP has been extended to multicast scenarios in some prior work, e.g., MZR [9] and MZRP [10]. However, MZP has the same problem as ZRP in query control. MZRP also utilizes the same

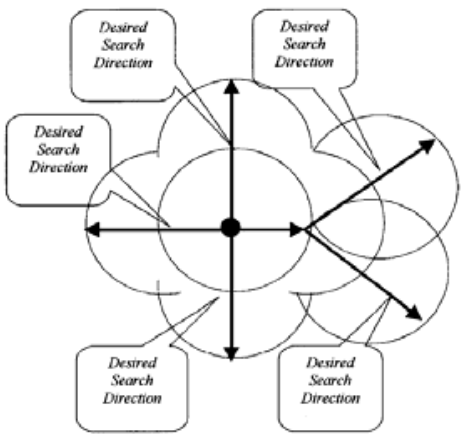

Figure 1. Guiding the route search along different directions.

method as ZRP to discover the route from a candidate group member to the multicast group. Therefore, both of them have not improved the route discovery mechanism in ZRP.

\section{PRELIMINARIES \\ 3.1 Geographic Partition}

With the ever-increasing advancement in location systems, mobile nodes can easily obtain their own positions with high accuracy by indoor or outdoor location technologies. In addition to being used for identifying mobile nodes' positions, the partition of the network coverage area has also been widely exploited in geographic routing [2]. It has been observed that geographic information can significantly improve the routing performance in MANETs. For ZRP route query, an ideal case is that the desired search along different direction passes through different nodes. If we partition the network coverage area into small zones and let each route search go through different zones, the duplicate route queries can be eliminated. Hence, geographic information can greatly help in the guidance of the route search.

We assume each node in the network is equipped with GPS devices and knows its own position. GMZRP uses geographic partition to help reduce the route discovery overhead, as will be shown in Section 4. It adopts a network center based partition method. The center of the network coverage area is roughly estimated at the time the MANET is initialized. The partition starts at the network center and spreads outward. As will be shown in Figure 2, the area is partitioned into equal circle-shaped zones. As the dotted lines show, each circle contains a hexagon with the side length equal to the radius of the circle. These hexagons are non-overlapping but can completely cover the entire network. Thus, there is a central zone at the network center and many other zones spread around the central zone symmetrically.

Each zone has a unique zone ID. Each zone has six neighboring zones since a hexagon has six sides. We denote the radius of the circle as $R, R=0.5^{*} r$, where $r$ is the radio transmission range of the mobile nodes. As a result, each node is the direct neighbor of any other node within the same zone. At start-up, all nodes know the network center and the partition method. Hence, each node knows its own zone ID and any other node's zone ID given that node's position. 


\subsection{Data Structures}

The primary fields of the MRREQ packet used in GMZRP are as $<$ Broadcast_ID, s, G, Source_Zone, Hop_Cnt>. The Broadcast_ID, together with the source node's ID $\mathbf{s}$ and multicast group address G, uniquely identifies each MRREQ packet. The Broadcast_ID is incremented for each MRREQ packet the source initiates for the same group. The Hop_Cnt is initialized by $\mathbf{s}$ to 0 and is incremented by each node forwarding the packet. The primary fields of the MRREP packet used by multicast forwarding tree establishment are as $<$ Broadcast_ID, s, G, r, Receiver_Zone, Zone_ID_Chain>. The Zone_ID_Chain records the IDs of the zones that the MRREP packet has passed and the latest one is placed at the beginning of the chain.

\section{MULTICAST TREE ESTABLISHMENT}

Multicast sources and receivers using GMZRP cooperate to establish and maintain forwarding state in the network to allow multicast communication. In GMZRP, the multicast forwarding state for a given multicast group $\mathbf{G}$ and source $\mathbf{s}$ is conceptually represented as a loosely-structured multicast forwarding tree rooted at $\mathbf{s}$. Each multicast packet is dynamically forwarded from $\mathbf{s}$ through the tree to the receiver members of the multicast group G.

In GMZRP, source-based multicast forwarding trees are created whenever there is at least one source and one receiver in the network. GMZRP is designed to work independently of the geographic unicast protocol used in the network and can thus work with any geographic unicast protocol. GMZRP currently operates only over bidirectional links.

\subsection{MRREQ Propagation}

A MRREQ packet, is initiated by a source node $\mathbf{s}$ that has data packets for G but no a Source Table entry for this multicast group. In this case, node $\mathbf{s}$ creates and initializes a new Source Table entry for $\mathbf{G}$. The source $\mathbf{s}$ then waits for replies in the form of MRREP packets from the receivers. If no reply is received from one or more receivers after a waiting period, $\mathbf{s}$ sends a new MRREQ. To avoid congesting the network, sending of the MRREQ packet is separated by an increasing interval using binary exponential backoff [12]. If the source still cannot receive any reply after a specified number of retries, the upper layer is informed that $\mathbf{G}$ is not reachable.

Once $\mathbf{s}$ receives at least one MRREP packet, $\mathbf{s}$ then begins sending normal multicast packets. However, it is possible that some interested receivers did not receive this initial MRREQ packet from $\mathbf{s}$ or some other receivers wish to leave the group $\mathbf{G}$ after a certain time. To allow for such occurrences caused by dynamic group membership, node $\mathbf{s}$ will rebroadcast the same MRREQ packet with incremented Broadcast_ID after a period of time. The time between each MRREQ broadcast is increased until reaching a slow background rate, designed to tolerate factors such as intermittent wireless interference or temporary partition of the mobile ad hoc network. By means of this, the multicast tree structure might be refined in reaction to membership change. Stale routes may be purged and new ones created.

In most prior work $[8,11,13]$, the route request was flooded in the network, which incurs high overhead. For ZRP, as introduced in Section 2, a few mechanisms based on topological information have been proposed to help reduce the duplicate queries. However, those solutions still have intrinsic problems since the network topology cannot provide effective information for guidance. Based on the above observations, GMZRP utilizes geographic partition to guide the MRREQ propagation. Clearly, the ideal case is that each zone is to be queried only once.

As shown in Figure 2, by our partition method the zones are distributed around the network center symmetrically. We mark the central zone as the first layer zone, the 6 zones neighboring to the first layer zone as the second layer zones, and the 12 zones neighboring to the second layer zones as the third layer zones, and so on. Based on the symmetrical zone partition, a simple but effective strategy has been developed to avoid duplicate queries during the MRREQ packet propagation. In the following, we firstly describe and illustrate the strategy in a network covered by three layers of zones as shown by Figure 2. Then we derive the formal strategy used in a more general case where the network is covered by zones of $n$ layers.

Assume that the source node $\mathbf{s}$ is residing in the central zone, which is hence claimed as the source zone. It firstly forwards the MRREQ packet to each layer-2 zone using geographic forwarding where the center of each zone is the destination. Then each layer2 zone needs to further forward the MRREQ packet to layer-3 zones. How to avoid the case that two layer- 2 zones forward the

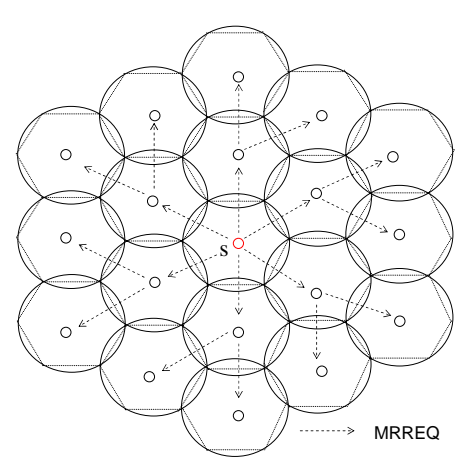

(a) From the first layer source zone

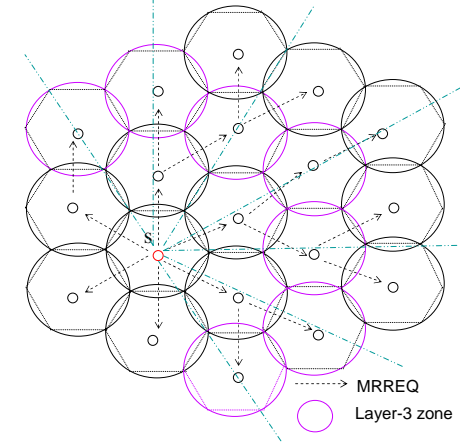

(b) From the second layer source zone

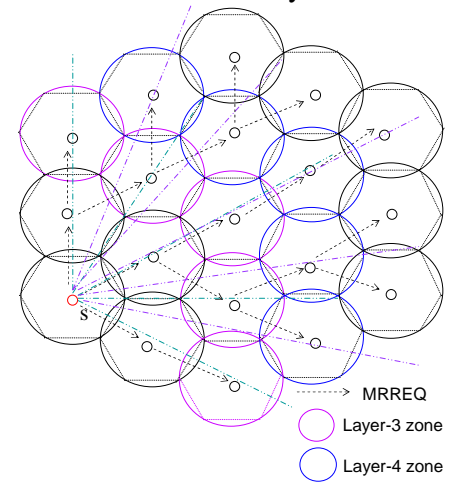

(c) From the third layer source zone

Figure 2. The propagation of the MRREQ packets originated from zones staying at different layers. 
same MRREQ packet to the same layer-3 zone, i.e., duplicate queries? Firstly, we draw a line to connect the center of the source zone and the center of a layer- 2 zone. We let the line pass through layer- 3 zones and clearly each such line passes through only one layer- 3 zone. Because the number of layer- 3 zones is double the number of layer-2 zones, each layer- 2 zone will forward the MRREQ packet to two layer- 3 zones: the zone the line passes and this zone's neighboring zone in clockwise direction. As shown in Figure 2(a), there is no duplicate query in the network.

If the source node $\mathbf{s}$ resides in one layer- 2 zone with respect to the network center, this zone becomes the source zone and we regard this source zone as the first layer zone during the propagation. Then we can see that there are three additional layers of zones around the source zone. Benefited from the partition, no matter where the source zone is, the zone partition covering the network is still part of a symmetrical zone partition around the current source zone. Hence, the above mentioned strategy of MRREQ packet propagation from layer-2 zones to layer-3 zones still applies. But, we need to decide how the layer-3 zones forward the MRREQ packets to layer- 4 zones. The method is similar. We firstly draw a line to connect the center of the source zone and the center of a layer- 3 zone. We let this line pass through layer- 4 zones. Then there are two different cases: one is that the line passes through only one layer- 4 zone and the other is that the line passes through the intersection part of two neighboring layer-4 zones. For the first case, the layer-3 zone will forward the MRREQ packet to that layer-4 zone only; and for the second case, the layer-3 zone will forward the MRREQ packet to those two layer-4 zones, respectively. As shown in Figure 2(b), there is also no duplicate query in the network.

Finally, if the source node $\mathbf{s}$ is residing in a layer- 3 zone with respect to the network center, accordingly this zone becomes the source zone, which is also regarded as the first layer zone during the propagation. We can see that there are four additional layers of zones around the source zone. The above mentioned strategy of MRREQ packet propagation from layer-3 zones to layer-4 zones still applies. What remains is that we need to decide how the layer- 4 zones forward the MRREQ packets to the layer- 5 zones. The method is exactly the same as the one used from layer- 3 zones to layer- 4 zones. We firstly draw a line to connect the center of the source zone and the center of a layer-4 zone. We let this line pass through layer-5 zones. Then there are also two different cases: one is that the line passes through only one layer5 zone and the other is that the line passes through the intersection part of two neighboring layer-5 zones. For the first case, the layer- 4 zone will forward the MRREQ packet to that layer- 5 zone only; and for the second case, the layer- 4 zone will forward the MRREQ packet to those two layer-5 zones, respectively. As shown in Figure 2(c), there is also no duplicate query in the network.

From the above description, we can see that an intermediate zone makes the MRREQ forwarding decision based on both its own position and the source zone's position. We derive the following rules, which build up the strategy of the MRREQ packet propagation. Here, the source zone is denoted as the first layer zone.

a) A layer-1 zone forwards a MRREQ packet to each layer-2 zone, separately; b) A layer-2 zone respectively forwards a MRREQ packet to two layer-3 zones if both of them exist. These two layer- 3 zones are the one passed by the line connecting the center of the source zone and the center of the corresponding layer-2 zone, and its neighboring zone in clockwise direction.

c) A layer-3 zone forwards a MRREQ packet to one or two layer- 4 zones if they exist. The one or two layer- 4 zones are the one(s) passed by the line connecting the center of the source zone and the center of the corresponding layer-3 zone.

d) A layer-4 zone forwards a MRREQ packet to one or two layer-5 zones if they exist. The one or two layer-5 zones are the one(s) passed by the line connecting the center of the source zone and the center of the corresponding layer-4 zone.

We then derive the following common rule, which can be proved by the symmetry properties of our partition method. Here the proof is omitted due to space limit.

e) For $n(n>2)$, a layer- $n$ zone forwards a MRREQ packet to one or two layer- $(n+1)$ zones if they exist. The one or two layer- $(n+1)$ zones are the one(s) passed by the line connecting the center of the source zone and the center of the corresponding layer- $n$ zone.

In addition, the proposed strategy can guarantee that the MRREQ packets reach all the network nodes with minimum delay. This property is also illustrated in Figure 2. A MRREQ packet originated at the source zone (i.e., layer- 1 zone) needs $n-1$ times of inter-zone forwarding before it reaches one layer- $n$ zone, which is just the shortest distance at the zone level. The detailed proof is also omitted due to space limit.

\subsection{MRREP Propagation}

As the MRREQ packet is broadcast across the network, nodes set up pointers to establish the reverse path. When a node $\mathbf{r}$ receives a MRREQ packet and it is an interested receiver, then $\mathbf{r}$ replies with a MRREP packet, to cause the necessary nodes along the path back to the source $\mathbf{s}$ to become forwarding nodes. $\mathbf{r}$ also creates a new entry in its Membership Table and sets the receiver flag. The MRREP packet then follows the path established by the forwarding of the received MRREQ packet, as recorded in the previous hop address field in each node's Node Table entry for this source $\mathbf{s}$. When the MRREP packet enters a new zone, the new zone ID will also be recorded into the packet and thus a zone ID chain will be formed and gradually expanded. Each node that forwards the MRREP packet, if it does not already have a Membership Table entry for this group and source, creates a new entry setting the forwarder flag and recording all its child nodes from which it has received appropriate MRREP packets. The new entry also records the current zone ID chain carried by the MRREP packet. When the source receives an appropriate MRREP packet, it will record the node ID and zone ID of the child from which it has received this MRREP packet. It also records the current zone ID chain carried by the MRREP packet.

\section{PERFORMANCE EVALUATION}

We have evaluated the performance of GMZRP through detailed packet-level simulation in a variety of mobility and communication scenarios. In addition, we have simulated the ODMRP, the best-studied on-demand multicast protocol for 
MANETs. ODMRP periodically floods the network with a control packet to re-create the multicast forwarding state. It allows redundant forwarding to each receiver, and hence increases the packet delivery ratio. We compare the obtained performance of GMZRP with that of ODMRP.

\subsection{Simulation Environment}

In order to evaluate the performance of GMZRP, we implement and simulate it in Glomosim [17]. We use 802.11 MAC protocol with DCF and a transmission range of $250 \mathrm{~m}$. Geographic forwarding adopts GPSR with activated perimeter mode. Nodes follow the Random Waypoint mobility model, where each node moves at a constant speed chosen randomly from a predefined speed range. The speed range is different for different simulation scenario. In each simulation run, we simulate the behaviour of 100 nodes in a $1.2 \mathrm{~km} \times 1.2 \mathrm{~km}$ square, which can be partitioned into 19 full zones as shown in Figure 2. The simulation time is 100 seconds. The multicast sources in our simulations generate constant bit rate (CBR) traffic, with each source originating 5128 bytes packets per second. Here, we only consider multicasting with single source. Each simulation scenario is repeated for 10 times and the average results are obtained.

In these experiments, we assume two types of networks: quasistatic ad hoc networks and mobile ad hoc networks. In the mobility model followed by quasi-static ad hoc networks, the pause time is set to be 50 seconds and the node speed range is $\left[0 \mathrm{~ms}^{-1}, 5 \mathrm{~ms}^{-1}\right]$. Quasi-static ad hoc networks simulate the scenario that nodes stay stationary or move slowly. In the mobility model followed by mobile ad hoc networks, the pause time is 0 seconds and the node speed range is set to be $\left[15 \mathrm{~ms}^{-1}, 20 \mathrm{~ms}^{-1}\right]$. A pause time of 0 represents a network in which all nodes move continuously. We also vary the multicast group size in different experiments.

\subsection{Simulation Results}

The commonly used performance metrics that we are also interested in are:

- Packet delivery ratio: The ratio of total number of packets received by all the receivers to the total number of packets originated by the source times the number of receivers.

- Normalized packet overhead: The total number of control and data packets transmitted by any node in the network (either originated or forwarded), divided by the total number of data packets received across all multicast receivers. This metric represents the total packet overhead normalized by the total received packets.

Figure 3 shows the comparison results on packet delivery ratio under different multicast group sizes. In the quasi-static network, both GMZRP and ODMRP achieve the packet delivery ratio over $97.8 \%$ for all multicast group sizes and they have competing performance. In the mobile network, ODMRP outperforms GMZRP slightly by delivering within $1 \%$ of the multicast data packets due to redundant forwarding. However, ODMRP has contributed more than 3 times protocol overhead to this minor improvement compared to GMZRP. In addition, by comparing Figures 3(a) and 3(b), we can see that GMZRP works a little better in quasi-static network than in mobile network. It means

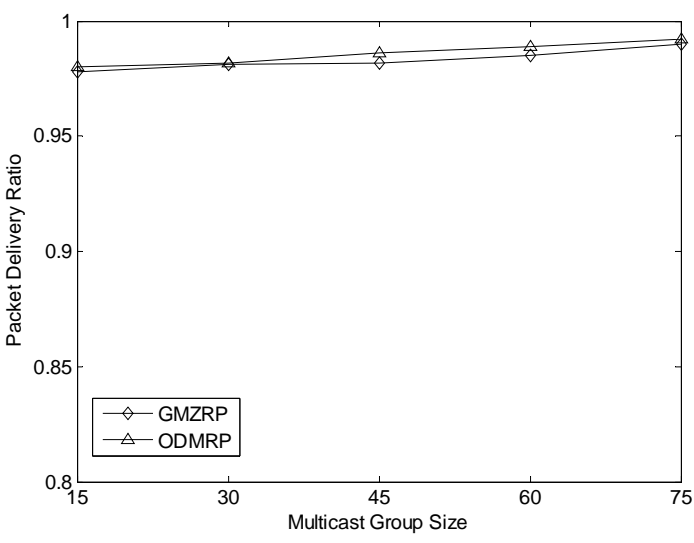

(a) Results in quasi-static networks

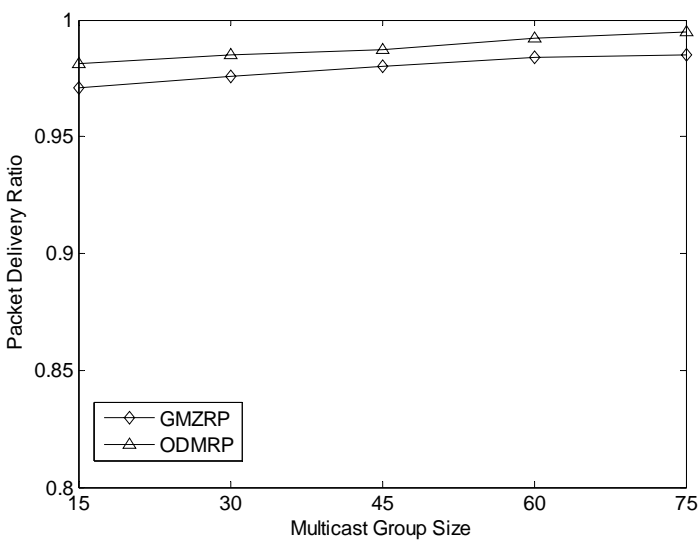

(b) Results in mobile networks

Figure 3. The comparison results in the packet delivery ratio between GMZRP and ODMRP.

that node mobility will affect the performance of GMZRP but very slightly.

In GMZRP, the primary control packet overhead comes from the propagation of MRREQ packets. With the increase in multicast group size, more receivers will reply a MRREP packet to the source. However, due to MRREP filtering at intermediate forwarding nodes, the increase in transmissions of MRREP packets is trivial. The number of other control packets is also negligible. Benefited from our strategy of MRREQ packet propagation, the number of MRREQ packet transmissions is independent of the multicast group size. Furthermore, the MRREQ packet propagation strategy is kind of broadcast technique instead of flooding. But in ODMRP, both control and data packets need to be flooded in the network periodically. Therefore, as shown in Figure 4, ODMRP has generated much higher packet overhead than GMZRP.

In addition, with the increase in multicast group size, a larger fraction of the nodes have established forwarding state, and the density of forwarding nodes is higher. This will help GMZRP create a more efficient multicast tree, through which the number of packet transmissions shared by multiple receivers is increased. 


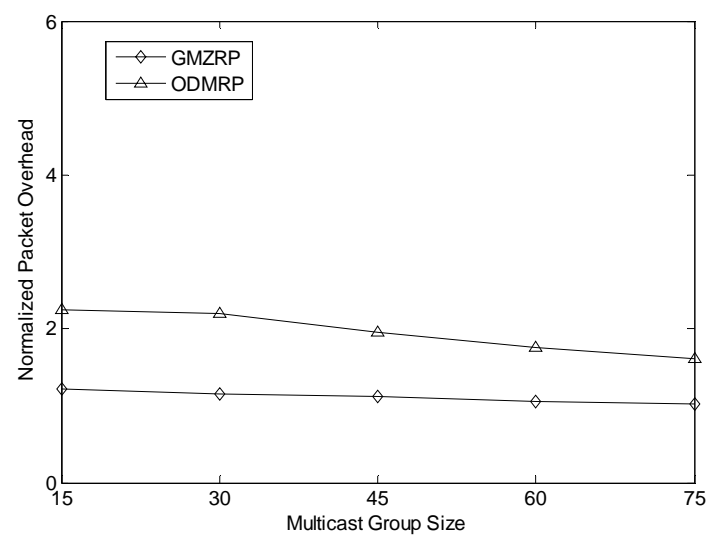

(a) Results in quasi-static networks

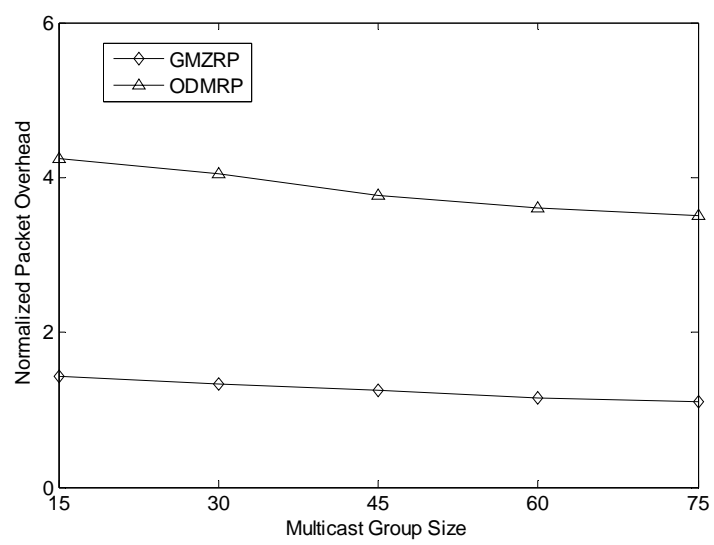

(b) Results in mobile networks

Figure 4. The comparison results in the normalized packet overhead between GMZRP and ODMRP.

Hence, in GMZRP, the normalized packet overhead will be decreased as the multicast group size increases. A larger multicast group also helps create a natural redundancy which ODMRP exploits through the flood forwarding of the multicast data packets within the forwarding nodes. Since the packet overhead presented here is normalized to the total number of received packets, we can see that both protocols show lower overhead with more receivers, as shown in Figure 4.

In addition, for both GMZRP and ODMRP, the overall overhead in lower mobility scenarios (quasi-static networks) is less than in the scenarios with high mobility (mobile networks). In GMZRP's case, this decrease is due to reduced broken links. In ODMRP's case, this is due to the creation of less redundant state.

\section{CONCLUSIONS}

In this paper, we introduce the Geography-aided Multicast Zone Routing Protocol (GMZRP) for multicast routing in mobile ad hoc networks. It is kind of hybrid routing, combining the advantages of both topological routing and geographic routing. It uses an efficient MRREQ propagation strategy to establish the multicast forwarding tree along the shortest paths and with the lowest overhead. In addition, each tree node also maintains a zone ID chain to each downstream receiver to benefit the recovery of broken links. Simulation results show that GMZRP has decent performance in terms of packet delivery ratio and normalized packet overhead.

\section{ACKNOWLEDGMENTS}

This work was supported in part by the University Grant Council of Hong Kong under the CERG grant PolyU 5170/03E, by the China National 973 Program Grant 2007CB307100.

\section{REFERENCES}

[1] C. Siva Ram Murthy and B.S. Manoj, Ad Hoc Wireless Networks: Architectures and Protocols, Prentice Hall PTR, 2004.

[2] Lap Kong Law, Srikanth V. Krishnamurthy, and Michalis Faloutsos, "Fireworks: An Adaptive Group Communications Protocol for Mobile Ad Hoc Networks," Proc. IFIP Networking, pp. 853-868, 2005.

[3] C. M. Cordeiro, H. Gossain, and D. P. Agrawal, "Multicast over Wireless Mobile Ad Hoc Networks: Present and Future Directions,” IEEE Network, Vol. 17, No. 1, pp. 52-59, 2003.

[4] S. Yang and J. Wu, "New Technologies of Multicasting in MANET," in Design and Analysis of Wireless Networks, Nova Science Publishers, 2005.

[5] H. Cheng and J. N Cao, "A Design Framework and Taxonomy for Hybrid Routing Protocols in Mobile Ad Hoc Networks”, IEEE Communications Surveys \& Tutorials, Vol. 10, No. 4, 2008.

[6] S. Giordano, I. Stojmenovic, and L. Blazevic, "Positionbased Routing Algorithms for Ad Hoc Networks: A Taxonomy," in Ad Hoc Wireless Networking, Norwell, MA: Kluwer, 2003.

[7] Zygmunt J. Hass and Marc R. Pearlman, "The Performance of Query Control Schemes for the Zone Routing Protocol," IEEE/ACM Transactions on Networking, Vol. 9, No. 4, pp. 427-438, 2001.

[8] S. Lee, W. Su, J. Hsu, M. Gerla, and R. Bagrodia, "A Performance Comparison Study of Ad Hoc Wireless Multicast Protocols," Proc. IEEE INFOCOM, 2000.

[9] Vijay Devarapalli and Deepinder Sidhu, "MZR: A Multicast Protocol for Mobile Ad Hoc Networks," Proc. ICC, 2001.

[10] X. Zhang and L. Jacob, "MZRP: An Extension of the Zone Routing Protocol for Multicasting in MANETs," Journal of Information Science and Engineering, Vol. 20, No. 3, pp. 535-551, 2004.

[11] Jorjeta G. Jetcheva and David B. Johnson, “Adaptive Demand-Driven Multicast Routing in Multi-hop Wireless Ad Hoc Networks," Proc. MobiHoc, 2001.

[12] Alvin C. Valera, Winston K.G. Seah, and S.V. Rao, "Improving Protocol Robustness in Ad Hoc Networks through Cooperative Packet Caching and Shortest Multipath Routing," IEEE Transactions on Mobile Computing, Vol. 4, No. 5, pp. 443-457, 2005.

[13] Ching-Chuan Chiang, Mario Gerla, and Lixia Zhang, "Forwarding Group Multicast Protocol (FGMP) for 
Multihop, Mobile Wireless Networks,” Cluster Computing, Vol. 1, No. 2, pp. 187-196, 1998.

[14] My T. Thai, Yingshu Li, and Ding-Zhu Du, "A Combination of Wireless Multicast Advantage and Hitch-hiking," IEEE Communications Letters, Vol. 9, No. 12, pp. 1037-1039, 2005.

[15] X. Du and D. Wu, "Adaptive Cell-Relay Routing Protocol for Mobile ad hoc Networks," IEEE Transactions on Vehicular Technology, Vol. 55, No. 1, pp. 278-285, 2006.
[16] Brad Karp and H. T. Kung, "GPSR: Greedy Perimeter Stateless Routing for Wireless Networks,” Proc. MobiCom, 2000.

[17] X. Zeng, R. Bagrodia, and M. Gerla, "GloMoSim: A Library for Parallel Simulation of Large-scale Wireless Networks," Proc. the 12th Workshop on Parallel and Distributed Simulations, 1998. 